\title{
Group Formation based on Students' Learning Styles by Circular Genetic Algorithm
}

\author{
Anon Sukstrienwong \\ School of Information Technology and Innovation, Bangkok University, Pathumthani, Thailand
}

\begin{abstract}
Student group formation plays a critical role in promoting academic learning styles. Therefore, in this study, we employed a heuristic algorithm called the circular genetic algorithm (CGA) to form balanced groups of dissimilar students in terms of personal preferred learning styles. The benefit of balancing students' learning styles is to assist students with diverse abilities to work collaboratively. With the assistance of our developed web application, members within a group contain mixed characteristics, while all formed groups of students are similar. In addition, an empirical case study was used to verify the feasibility and effectiveness of the proposed algorithm. Corresponding results verified by the analysis of variance (ANOVA) indicate that the algorithm can optimally allocate students in an efficient way.
\end{abstract}

Keywords - Circular Chromosome, Genetic algorithms, Group Formation, Learning styles, Web application

\section{Introduction}

Over the past several years, group formation has been successfully employed as an educational approach to arrange students into appropriate learning groups.

DOI: 10.18421/TEM103-02

https://doi.org/10.18421/TEM103-02

Corresponding author: Anon Sukstrienwong,

School of Information Technology and Innovation, Bangkok University, Pathumthani, Thailand.

Email: anon.su@bu.ac.th

Received: 23 March 2021.

Revised: 11 July 2021.

Accepted: 17 July 2021.

Published: 27 August 2021.

(c) BY-NC-ND (C) 2021 Anon Sukstrienwong; published by UIKTEN. This work is licensed under the Creative Commons Attribution-NonCommercial-NoDerivs 4.0 License.

The article is published with Open Access at www.temjournal.com
Building appropriate groups in a class is crucial to teamwork success [1]. Therefore, several approaches for solving the group formation of students related to the learning state of students are proposed. For example, Alfonseca et al. [2] explored the impact of learning styles on the grouping of students in collaborative tasks. The authors reported that some dimensions of the learning style model affect the quality of student work. Subsequently, Abuseileek [3] reported the effect of cooperative learning for a small group. The author used two instructional modes, positive interdependence and individual accountability, on English for Foreign Language (EFL) undergraduate learners' communication skills. A paper by Grigoriadou et al. [4] studied how learning styles and knowledge influence group collaboration. The authors also supported group formation modules, which could be incorporated in an adaptive educational system in order to support cooperative learning. Michael et al. [5] developed a system to help allocating a proper project topic to students based on their academic performance.

Jahanbakhsh et al. [6] addressed the fact that most students tend to choose teammates akin to them, which is called a homogenous group. This approach is common to team formation. However, it lacks the skill diversity needed to achieve the common goal. The authors also emphasized that teachers are increasingly applying criteria-based approaches to form groups of students. Consequently, there exist many approaches for group formation. As a result, many algorithms have been proposed in order to optimally form groups of students. An interesting algorithm was presented by Jagadish [7]. The author presented an approach based on K-NN clustering algorithm for the group formation of learners in a collaborative learning environment to ensure that all members' learning is optimized. Abnar et al. [8] constructed a mechanism for forming learning groups. Some data about students are extracted from their profiles in MOODLE before applying their GAbased algorithm. A method presented by Zheng et al. [9] focuses on building an optimal group formation based on various grouping requirements. In this work, an improved GA is implemented to mix and 
balance the numbers of characteristics for homogeneous and heterogeneous learning groups.

In this paper, we proposed a new genetic-algorithm approach for heterogeneous grouping, in consideration to the other aspect. Importantly, the circular chromosome is designed to accompany the group formation. Moreover, in this work, we adopt three main sensory learning styles, namely visual, auditory, and kinesthetic learning (VAK), which students use to receive information [10]. The VAK concepts were first introduced by psychologists and child teaching specialists such as Fernald, Keller, Orton, Gillingham, Stillman and Montessori, starting in the 1920's [11]. Due to the diversity of students in classes, forming an optimized group is becoming more complicated and somewhat time-consuming. Consequently, a web application is developed in order to verify efficiency of the proposed algorithm.

\section{Circular Chromosome Representation}

GAs are based on the evolutionary ideas of natural selection and genetics. These are easy to implement and very useful to solve optimization problems. Most GA-based approaches are represented in binary as strings of $0 \mathrm{~s}$ and $1 \mathrm{~s}$, but other encodings on designing the chromosome are also possible. There exist many techniques on designing the chromosome. In this paper, a cyclic chromosome is designed. Let $S=\left\{s_{1}, s_{2}, \ldots, s_{n}\right\}$ denote the set of $n$ students. We then encode the set of students who are different in preference of the VAK learning style into a chromosome. The circular chromosome used in this paper is illustrated in Figure 1. Genes lined up in the sequence of students are grouped together. The number of groups $(m)$ and number of members per groups $(p)$ must be specified before forming the groups. Each group $G_{i}, 1<i \leq m$ contains $p$ members.

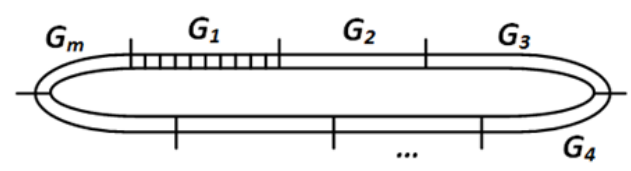

Figure 1. Circular Chromosome representation for group formation of $m$ groups

Fitness function: The fitness value of chromosomes is calculated in a way associated with the circular chromosome structure. As stated previously, our algorithm aims to balance the VAK learning style, with the average value of each group being calculated. Additionally, the Euclidean distance between all groups of three sensory receivers will be calculated. Then, the fitness function can be presented in (1), where $A v g_{V}$ is the average value of Visual learning style for all members, $A v g_{A}$ is the average value of Auditory learning style for all members, and $A v g_{K}$ is the average value of Kinesthetic learning style of all members.

Fitness $($ chromosome $)=$

$\sum_{i=1}^{m-1} \sum_{j=i+1}^{m} \sqrt{\begin{array}{c}\left(A v g_{V_{i}}-A v g_{V_{j}}\right)^{2}+\left(A v g_{A_{i}}-A v g_{A_{j}}\right)^{2} \\ +\left(A v g_{K_{i}}-A v g_{K_{j}}\right)^{2}\end{array}}$

, where $m$ is the number of groups.

Our circular chromosome is similar to a linear chromosome, but it has no terminal ends. This representation strongly affects the behaviour and operators of our proposed genetic algorithm. The breeding operators must also take into account the chromosome's design. Typically, the mutation operator and crossover operator must be designed to create the next generation. However, in this work, two new operators associated with the circular chromosomes are designed.

Circular-shift operator: The operator starts by randomly selecting a circular chromosome to participate. Then, it randomly chooses a number $(q)$, where $1<q<n$. All genes of the participated chromosome move clockwise $q$ times. An example of how the circular-shift operator works is demonstrated in Figure 2. The result of shifting the chromosome clockwise by $q$ times generates offspring, which forms a new coalition. As a result, all groups are changed.

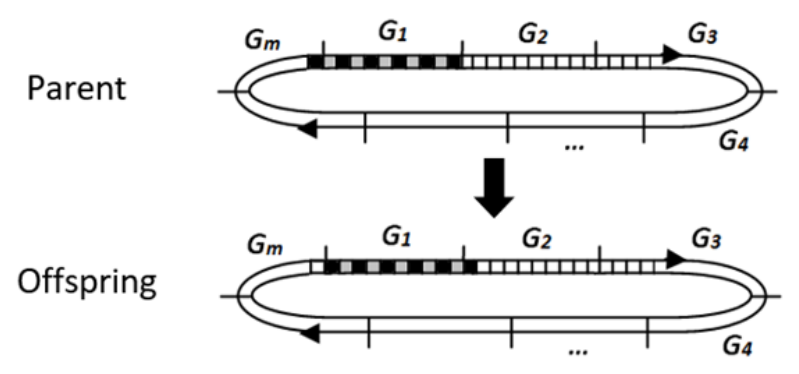

Figure 2. Circular-shift operator, $q=1$

Self-crossover operator: This operator is similar to the crossover operator for a linear chromosome. In general, crossover is done by combining portions from old ones to create new ones [12]. Self-crossover operator begins by swapping two random portions of a selected parent from the current population to generate an offspring for the next generation. Additionally, the crossover rate $\left(p_{c}\right)$ must be userdefined in order to control the number of crossovers that perform in one generation. A high crossover rate makes most of the population to perform the crossover, which is possible to encourage good mixing of the chromosomes. However, a high crossover rate may cause GA to often become trapped in a local minimum and fail to find better 
solutions. Figure 3. illustrates how the self-crossover operator of a circular chromosome performs to create a newborn chromosome. Everything between two portions is swapped. In order to do this, two portions must be equal in length.

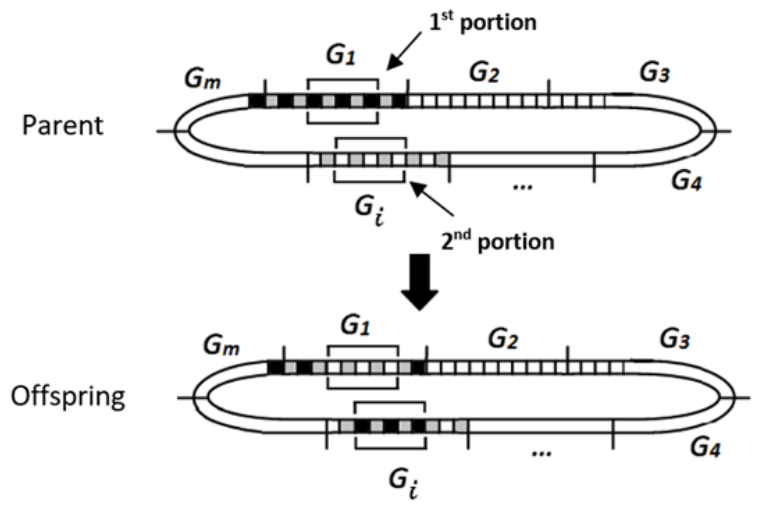

Figure 3. Self-crossover of two random portions creating a newborn chromosome

\section{System Design}

In the development of this system, system analysis and design are necessary. Hence, this section describes the web application development for Group Composition based on the VAK learning styles, referred as to "GroupUsVAK. The ultimate aim for this development is to facilitate teachers in the classes where VAK learning styles are used. We have developed the system using the following software:

- AppServ Version.8.4.0

- MySql Workbench

- XAMPP

Planning work before implementation will help to accomplish our goal, because if there is a problem it can be resolved effortlessly at the point. In addition, working in a systematic manner allows us to specify a definite deadline for program development, which is considered to be very useful in today's program development. In order to summarize the details of our system, the use case diagram is demonstrated in Figure 4. There are two kinds of users, teachers and students. Teachers are able to create a new class in the system. For students who want to join a specific class, they will need to have a joining code given to them by their teacher. Additionally, graphical user interfaces for users, teachers and students, and their relations between formation processes are presented in Figure 5.

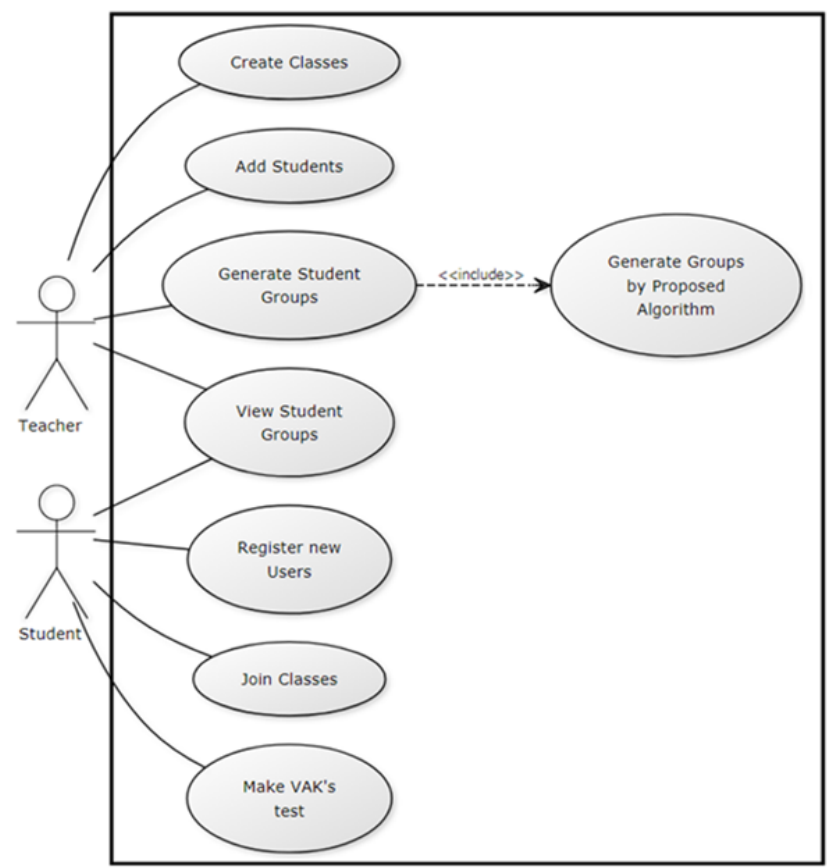

Figure 4. Use case diagram of GroupUsVAK

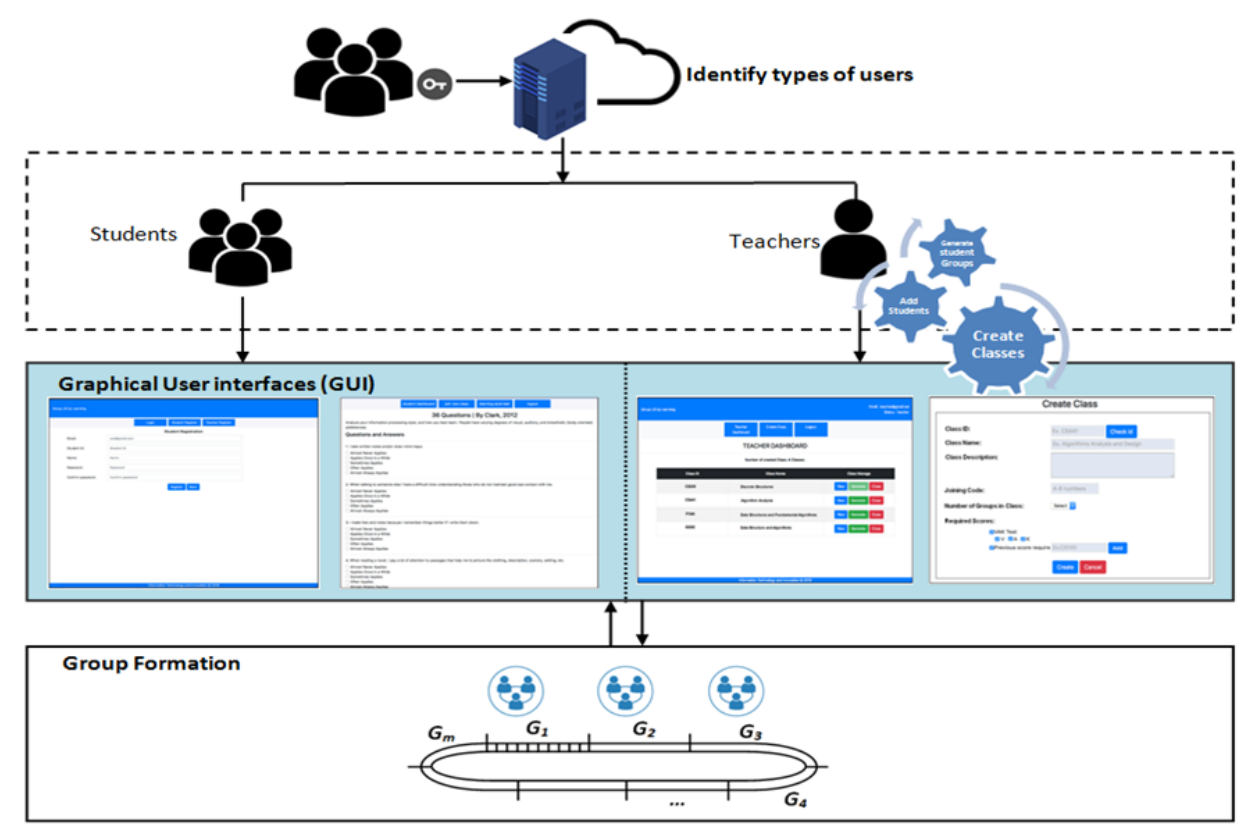

Figure 5. Information flow between actors, which are teachers and students 


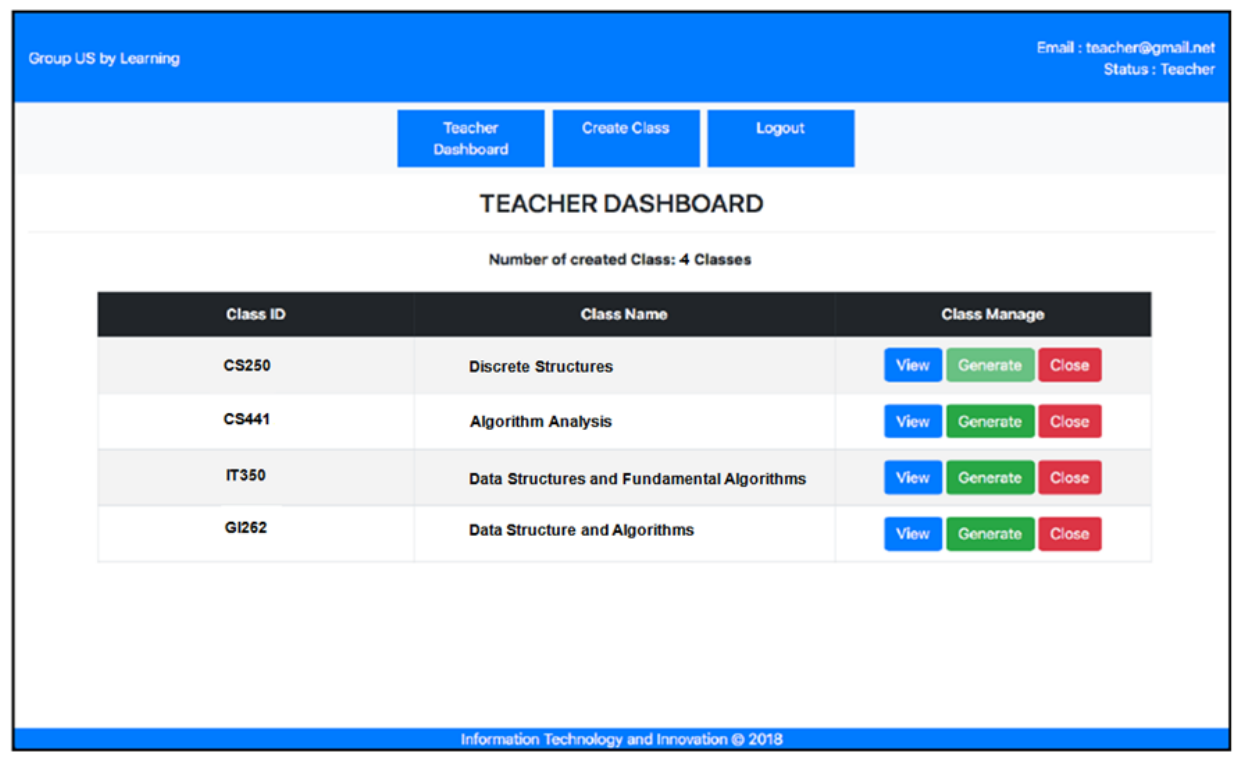

Figure 6. The example screenshot of the teacher dashboard

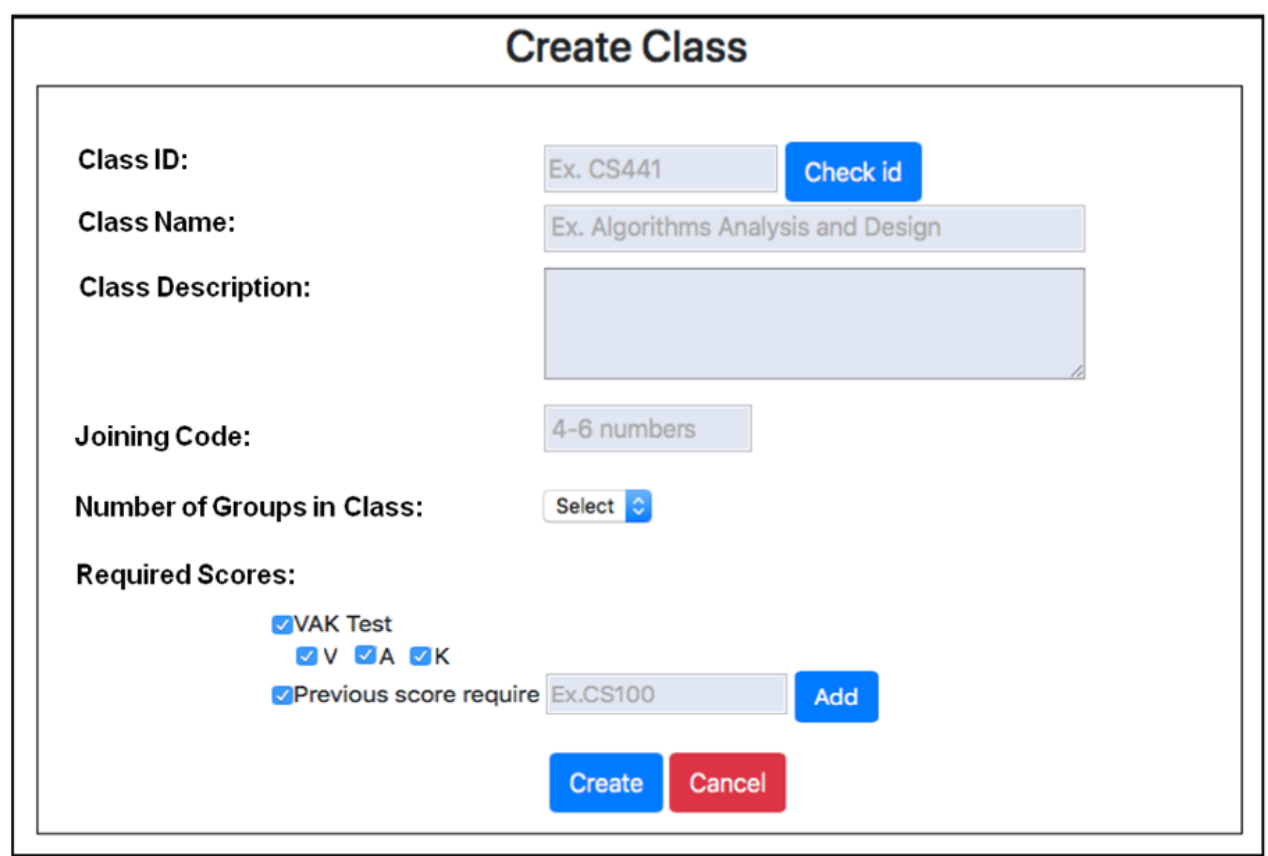

Figure 7. The example screenshot for creating a class

The screenshot examples for the teacher interface in creating a new class are presented in Figure 6. and Figure 7. The example screenshot of the Teacher Dashboard illustrated in Figure 6. details all available classes. It provides multiple features for the teachers. The 'View' button is for viewing the class details, class name, class description, number of students per group or number of groups, and number of registered students. After all students have finished joining the class, the teachers choose the 'Generate' button to allow the system to form the groups of students by our proposed genetic algorithm. The 'Close' button is for teachers to disable a given class on the GroupUsVAK after the completion of the class.
The teachers are able to create a new class containing class details and grouping criteria, such as class name, number of groups or number of students per group, scores required for forming groups, and joining code. At this point, the teachers can set the required previous scores and the VAK tests (the total 36 of question). Therefore, all registered students will be notified to take the VAK tests before processing the grouping. The example screenshot for students to take the VAK is presented in Figure 8. However, if any registered students have already made a VAK learning test, the system will automatically allow them to select the class that they are interested in participating in. 


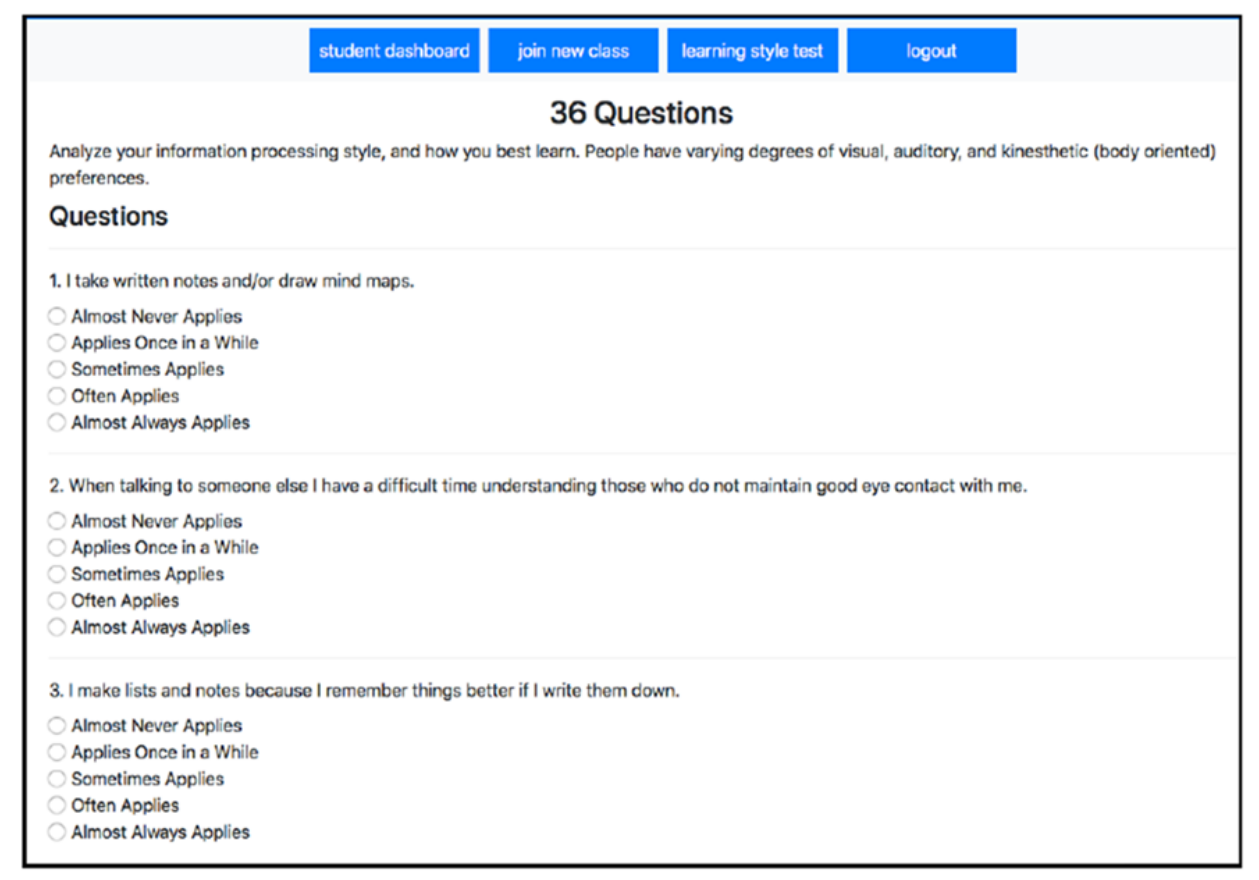

Figure 8. The example screenshot for students to take VAK test

\section{Performance Test}

In order to examine the efficiency of the algorithm, an empirical dataset of 100 students was used. Additionally, we determine the number of students per group arbitrarily equal to 5 . We use only VAK scores as the primary setting for forming groups. Figure 9. presents that the average VAK score of each group is obtained by the algorithm. It points out that the proposed algorithm performed the group formation in a satisfactory manner, as the average scores of generated groups are similar. Moreover, the results of a two-way ANOVA of generated groups are shown in Table 1. As we can see, the results of the analysis show that generated groups can be calculated with the F-test $=0.378$ and the $\mathrm{p}$-value for the test of groups being 0.992 , which is greater than $5 \%(\alpha)$. Hence, it is confirmed that all generated groups are similar. In addition, the F-test for learning styles is 0.256 . The p-value is equal to 0.774 , which is also greater than $5 \%(\alpha)$. Hence, learning styles have no difference. Obviously, there is no interaction between groups and learning styles, as we get the pvalue for the test of groups of 1.000 . This indicates that there is no significant interaction between groups and learning styles at the significance level of 0.05 .

\section{Conclusions and future work}

In this paper, we have proposed a new GA approach using circular chromosomes for encoding group formation based on student learning styles. The web application was implemented to facilitate teachers to collect students' information and manage the processes of forming student groups. In order to verify the feasibility and effectiveness of the proposed algorithm, an empirical case study was used. According to our empirical experiments, the results demonstrate that the algorithm is able to form groups of dissimilar students, while all formed groups are similar in terms of learning styles verified by ANOVA. For future research, we shall identify the user satisfaction level of our web application to investigate which factors hold the most influence. Also, we shall continue to examine students' performance generated by the algorithm associated to three main sensory learning styles. Finally, we will perform the model on a big dataset to verify the efficiency and robustness of the algorithm in a collaborative learning environment of the university.

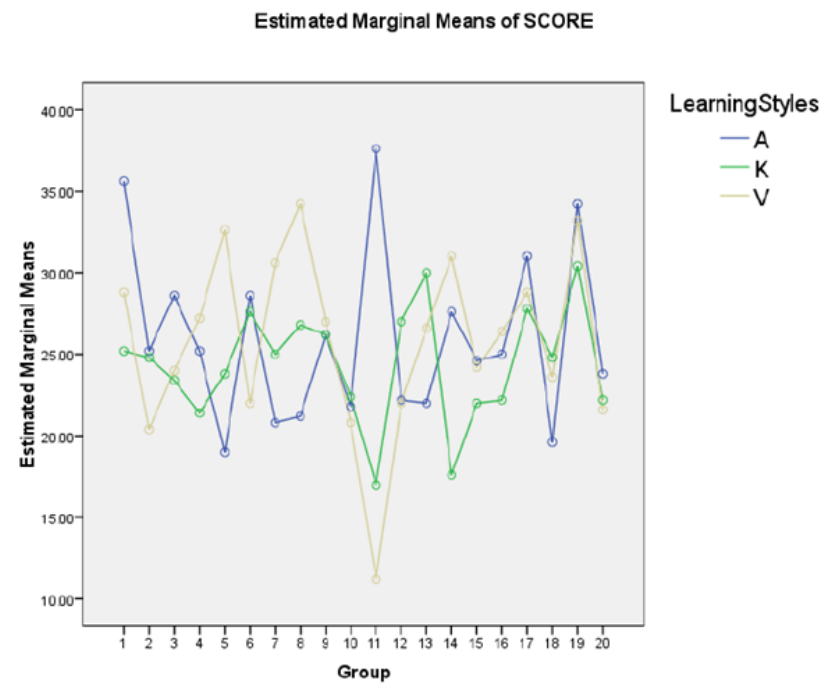

Figure 9. The average of each group of VAK scores 
Table 1. ANOVA's results

\begin{tabular}{|l|c|c|c|c|c|}
\hline \multicolumn{1}{|c|}{ Source } & $\begin{array}{c}\text { Type III Sum of } \\
\text { Squares }\end{array}$ & df & Mean Square & F & Sig. \\
\hline Corrected Model & $6844.787^{\mathrm{a}}$ & 59 & 116.013 & .382 & 1.000 \\
\hline Intercept & 193446.413 & 1 & 193446.413 & 636.522 & .000 \\
\hline Group & 2179.853 & 19 & 114.729 & .378 & 0.992 \\
\hline LearningStyles & 155.647 & 2 & 77.823 & .256 & .774 \\
\hline Group * LearningStyles & 4509.287 & 38 & 118.665 & .390 & 1.000 \\
\hline Error & 72938.800 & 240 & 303.912 & & \\
\hline Total & 273230.000 & 300 & & & \\
\hline Corrected Total & 79783.587 & 299 & & & \\
\hline
\end{tabular}

a. $\quad$ R Squared $=.086$ (Adjusted R Squared $=-.139$ )

\section{References}

[1]. Thanh, P. T. H., \& Gillies, R. (2010). Group Composition of Cooperative Learning: Does Heterogeneous Grouping Work in Asian Classrooms?. International Education Studies, 3(3), 12-19.

[2]. Alfonseca, E., Carro, R. M., Martín, E., Ortigosa, A., $\&$ Paredes, P. (2006). The impact of learning styles on student grouping for collaborative learning: a case study. User Modeling and User-Adapted Interaction, 16(3), 377-401.

[3]. AbuSeileek, A. F. (2012). The effect of computerassisted cooperative learning methods and group size on the EFL learners' achievement in communication skills. Computers \& Education, 58(1), 231-239.

[4]. Grigoriadou, M., Papanikolaou, K., \& Gouli, E. (2006, July). Investigating how to group students based on their learning styles. In Sixth IEEE International Conference on Advanced Learning Technologies (ICALT'06) (pp. 1139-1140). IEEE.

[5]. Michael, D. C., Cyprus, N., Kordal, N., \& Mukadi, B., (2020). Team Formation and Grouping Problem for Graduation Projects. The Online Journal of Quality in Higher Education, 7(1), 63-72.

[6]. Jahanbakhsh, F., Fu, W. T., Karahalios, K., Marinov, D., \& Bailey, B. (2017, May). You want me to work with who? Stakeholder perceptions of automated team formation in project-based courses. In Proceedings of the 2017 CHI conference on human factors in computing systems (pp. 3201-3212).
[7]. Jagadish, D. (2014, April). Grouping in collaborative e-learning environment based on interaction among students. In 2014 International Conference on Recent Trends in Information Technology (pp. 1-5). IEEE.

[8]. Abnar, S., Orooji, F., \& Taghiyareh, F. (2012, January). An evolutionary algorithm for forming mixed groups of learners in web based collaborative learning environments. In 2012 IEEE international conference on technology enhanced education (ICTEE) (pp. 1-6). IEEE.

[9]. Zheng, Y., Li, C., Liu, S., \& Lu, W. (2018). An improved genetic approach for composing optimal collaborative learning groups. Knowledge-Based Systems, 139, 214-225.

[10]. Ibrahim, R. H., \& Hussein, D. A. (2016). Assessment of visual, auditory, and kinesthetic learning style among undergraduate nursing students. Int J Adv Nurs Stud, 5(1), 1-4.

[11]. Gholami, S., \& Bagheri, M. S. (2013). Relationship between VAK learning styles and problem solving styles regarding gender and students' fields of study. Journal of Language Teaching and Research, 4(4), 700-707.

[12]. Abdoun, O., \& Abouchabaka, J. (2012). A comparative study of adaptive crossover operators for genetic algorithms to resolve the traveling salesman problem. arXiv preprint arXiv:1203.3097. 